\title{
THE CHOICE OF THE SPHERICAL RADIAL BASIS FUNCTIONS IN LOCAL GRAVITY FIELD MODELING
}

\author{
R. TENZER AND R. KLEES
}

Faculty of Aerospace Engineering, Physical and Space Geodesy (PSG), Delft University of Technology, Kluyverweg 1, 2629 HS Delft, The Netherlands (R.Tenzer@tudelft.nl)

Received: May 28, 2007; Revised: November 5, 2007; Accepted: November 20, 2007

\begin{abstract}
The choice of the optimal spherical radial basis function (SRBF) in local gravity field modelling from terrestrial gravity data is investigated. Various types of SRBFs are considered: the point-mass kernel, radial multipoles, Poisson wavelets, and the Poisson kernel. The analytical expressions for the Poisson kernel, the point-mass kernel and the radial multipoles are well known, while for the Poisson wavelet new closed analytical expressions are derived for arbitrary orders using recursions. The performance of each $S R B F$ in local gravity field modelling is analyzed using real data. A penalized leastsquares technique is applied to estimate the gravity field parameters. As follows from the analysis, almost the same accuracy of gravity field modelling can be achieved for different types of the SRBFS, provided that the depth of the SRBFs is chosen properly. Generalized cross validation is shown to be a suitable technique for the choice of the depth. As a good alternative to generalized cross validation, we propose the minimization of the RMS differences between predicted and observed values at a set of control points. The optimal regularization parameter is determined using variance component estimation techniques. The relation between the depth and the correlation length of the SRBFs is established. It is shown that the optimal depth depends on the type of the SRBF. However, the gravity field solution does not change significantly if the depth is changed by several $\mathrm{km}$. The size of the data area (which is always larger than the target area) depends on the type of the $S R B F$. The point-mass kernel requires the largest data area.
\end{abstract}

Key words: local gravity field modelling, penalized least-squares, spherical radial basis functions, variance component estimation, generalized cross validation

\section{INTRODUCTION}

Spherical radial basis functions (SRBFs) are a powerful parameterization for local gravity field modelling. The simplest example is the point-mass kernel, which has been proposed by Weightmann (unpublished results). Since then, gravity field modelling by point masses were addressed in many studies, e.g., Hardy and Göpfert (1975), Reilly and Herbrechtsmeier (1978), Heikkinen (1981), Sünkel (1981, 1983), Vermeer (1992, 1995), Blaha et al. (1986) and Lehmann (1993, 1995). Marchenko (1998) introduced the radial multipoles, which are higher-order radial derivatives of the point-mass kernel. Chambodut 
et al. (2005), Panet et al. (2006), and Klees and Wittwer (2007) use Poisson wavelets, which were introduced in Holschneider et al. (2003).

Whereas the Poisson wavelets, the point-mass kernel and the radial multipoles belong to the class of non-bandlimited SRBFs, Schmidt et al. $(2005,2007)$ use the band-limited Blackman basis functions. Among the SRBFs without closed analytical expressions, the band-limited property of the Blackman basis functions is an advantage, because of the reduced numerical complexity. Nevertheless, SRBFs in analytical form are preferred in local gravity field modelling due to the large number of data to be processed.

Freeden et al. (1998) introduced several scaling functions, which belong to the class of SRBFs and thus can be used in local gravity field modelling. Finally, numerous studies were addressed to the use of reproducing kernels in least-squares collocation, e.g., Lelgemann (1981), Tscherning (1986), Lelgemann and Marchenko (2001), and Sansò and Tscherning (2003). Most of them can be used directly as SRBFs in local gravity field modelling after applying the Kelvin transformation.

Although many types of SRBFs have been proposed in literature and applied to local gravity field modelling, a comparison of the performance of the most popular choices has not been done yet. Therefore, the first objective of the paper is to investigate the performance of various types of SRBFs in local gravity field modelling using real data. Moreover, the relation between the optimal depth of the SRBF and the correlation length is established. This relation is very useful in gravity field modelling as it is the correlation length, which can be directly related to the signal variation and the data density whereas the optimal depth depends on the type of SRBF. Moreover, the correlation length determines the size of the area where gravity data is needed to reduce edge effects. Finally, the performance of RMS minimization as alternative to general cross validation (GCV) for the choice of the optimal depth is investigated. RMS minimization is easy to implement and numerically more efficient than GCV in particular for large data sets.

The paper is organized as follows. In Section 2, the functional model and the representation of the SRBFs in the spatial and spectral domain are recapitulated. In Section 3, a sketch of the estimation principle, the observation-group weighting, and the regularization is given as far as needed for the numerical experiment addressed in Section 5; for more details about the local gravity field modelling process, we refer to Klees et al. (2008). The relation between the depth and the correlation length for different types of SRBFs is the subject of Section 4. In the numerical experiments of Section 5, various types of the SRBFs are adopted. The choice of the optimal depths of the SRBFs is investigated using real data. The method for the optimal depth selection using GCV is briefly summarized. GCV and RMS minimization techniques are then utilized for the optimal depth selection for different types of the SRBFs and the results are compared. Section 6 summarizes the main results.

\section{FUNCTIONAL MODEL}

We consider a residual gravity field, which is obtained after subtracting a global gravity field model, and the effect of topography and atmosphere (remove-restore technique). The corresponding residual disturbing gravity potential $T$ is approximated by a function, which is harmonic outside a Bjerhammar sphere. We call this function again 
residual disturbing gravity potential $T$ to keep the notation simple (Runge-Krarup theorem, Krarup, 1969). We express $T$ at a point $\boldsymbol{x}$ as a linear combination of $I$ spherical radial basis functions $\left\{\Psi\left(\boldsymbol{x}, \boldsymbol{y}_{i}\right): i=1, \ldots, I\right\}$ :

$$
T(\boldsymbol{x})=\sum_{i=1}^{I} \beta_{i} \Psi\left(\boldsymbol{x}, \boldsymbol{y}_{i}\right),
$$

where the coefficients $\left\{\beta_{i}: i=1, \ldots, I\right\}$ are to be determined from gravity data. After linearization and spherical approximation, the residual surface gravity anomalies $\Delta g$ and the residual gravity disturbances $\delta g$ are related to the residual disturbing potential according to the well-known formulae

$$
\Delta g(\boldsymbol{x}) \cong-\frac{2}{|\boldsymbol{x}|} T(\boldsymbol{x})-\frac{\partial T(\boldsymbol{x})}{\partial|\boldsymbol{x}|}, \quad \delta g(\boldsymbol{x}) \cong-\frac{\partial T(\boldsymbol{x})}{\partial|\boldsymbol{x}|} .
$$

In this paper, a SRBF is a function $\Psi$ at pole position $\boldsymbol{y}$ defined through (e.g., Klees et al., 2008)

$$
\Psi(\boldsymbol{x}, \boldsymbol{y})=\sum_{n=0}^{\infty} \psi_{\mathrm{n}}(\boldsymbol{y}) \frac{2 n+1}{R}\left(\frac{R}{|\boldsymbol{x}|}\right)^{n+1} P_{n}\left(\hat{\boldsymbol{x}}^{T} \hat{\boldsymbol{y}}\right), \quad|\boldsymbol{y}|<R, \quad|\boldsymbol{x}| \geq R,
$$

where $P_{n}$ is the Legendre polynomial of degree $n, \hat{\boldsymbol{x}}=\boldsymbol{x} /|\boldsymbol{x}|$ and $\hat{\boldsymbol{y}}=\boldsymbol{y} /|\boldsymbol{y}|$ are unit vectors in the direction $\boldsymbol{x}$ and $\boldsymbol{y}$, respectively, $\psi_{n}$ is the Legendre coefficient of degree $n$, and $R$ is the radius of the Bjerhammar sphere. The choice of the Legendre coefficients $\left\{\psi_{n}\right\}$ determines the type of the SRBF. The distance $d:=R-|y|$ is called the depth of the SRBF.

The point-mass kernel $\Psi_{p m}$ is defined by

$$
\psi_{n}=\frac{1}{2 n+1} \lambda^{n}, \quad \lambda=\frac{|\boldsymbol{y}|}{R} .
$$

After inserting Eq.(4) into Eq.(3), we arrive at

$$
\Psi_{p m}(\boldsymbol{x}, \boldsymbol{y})=\frac{1}{|\boldsymbol{y}|} \sum_{n=0}^{\infty}\left(\frac{|\boldsymbol{y}|}{|\boldsymbol{x}|}\right)^{\mathrm{n}+1} P_{n}\left(\hat{\boldsymbol{x}}^{T} \hat{\boldsymbol{y}}\right) .
$$

The corresponding spatial representation of the point-mass kernel $\Psi_{p m}$ equals the reciprocal Euclidean distance $|\boldsymbol{x}-\boldsymbol{y}|$, i.e.,

$$
\Psi_{p m}(\boldsymbol{x}, \boldsymbol{y})=\frac{1}{|\boldsymbol{x}-\boldsymbol{y}|} .
$$

The (scaled) radial derivatives of the point-mass kernel, 


$$
\Psi(\boldsymbol{x}, \boldsymbol{y})=(\partial / \partial|\boldsymbol{y}|)^{m}(m !|\boldsymbol{x}-\boldsymbol{y}|)^{-1},
$$

are known as radial multipoles of order $m$ (cf. Marchenko, 1998); the Legendre coefficients are

$$
\psi_{n}=\delta_{0,1}\left(\begin{array}{l}
n \\
m
\end{array}\right) \frac{1}{2 n+1} \lambda^{n-m},
$$

where $\delta_{0,1}=1$ for $n \geq m$, and $\delta_{0,1}=0$ for $n<m$. From Eqs.(3) and (7), the spectral representation of the radial multipoles $\Psi_{r m}^{(m)}$ reads

$$
\Psi_{r m}^{(m)}(\boldsymbol{x}, \boldsymbol{y})=\sum_{n=0}^{\infty} \delta_{0,1}\left(\begin{array}{l}
n \\
m
\end{array}\right) \frac{1}{|\boldsymbol{x}|}\left(\frac{R}{|\boldsymbol{y}|}\right)^{m}\left(\frac{|\boldsymbol{y}|}{|\boldsymbol{x}|}\right)^{n} P_{n}\left(\hat{\boldsymbol{x}}^{T} \hat{\boldsymbol{y}}\right) .
$$

Realizing that the zero-order radial multipole is equal to the point-mass kernel, i.e., $\Psi_{r m}^{(0)} \equiv \Psi_{p m}$, and the first-order radial multipole reads

$$
\Psi_{r m}^{(1)}(\boldsymbol{x}, \boldsymbol{y})=-\frac{|\boldsymbol{y}|-|\boldsymbol{x}|\left(\hat{\boldsymbol{x}}^{T} \hat{\boldsymbol{y}}\right)}{|\boldsymbol{x}-\boldsymbol{y}|^{3}},
$$

the radial multipoles of order $m \geq 2$ can be computed recursively (Marchenko, 1998),

$$
\begin{gathered}
\Psi_{r m}^{(m)}(\boldsymbol{x}, \boldsymbol{y})=(2 m-1)|\boldsymbol{x}-\boldsymbol{y}| \Psi_{r m}^{(1)}(\boldsymbol{x}, \boldsymbol{y}) \Psi_{r m}^{(m-1)}(\boldsymbol{x}, \boldsymbol{y}) \\
-(m-1)^{2} \Psi_{r m}^{(0)}(\boldsymbol{x}, \boldsymbol{y}) \Psi_{r m}^{(m-2)}(\boldsymbol{x}, \boldsymbol{y}), \quad m \geq 2 .
\end{gathered}
$$

The Poisson kernel $\Psi_{p k}$ is defined by

$$
\psi_{n}=R \lambda^{n+1}
$$

From Eqs.(3) and (11), we get

$$
\Psi_{p k}(\boldsymbol{x}, \boldsymbol{y})=\sum_{n=0}^{\infty}(2 n+1)\left(\frac{|\boldsymbol{y}|}{|\boldsymbol{x}|}\right)^{n+1} P_{n}\left(\hat{\boldsymbol{x}}^{T} \hat{\boldsymbol{y}}\right) .
$$

The spatial representation of $\Psi_{p k}$ reads

$$
\Psi_{p k}(x, y)=|y| \frac{|x|^{2}-|y|^{2}}{|x-y|^{3}} .
$$

Finally, the Poisson wavelets $\Psi_{p w}^{(m)}$ of order $m$ are defined as (Holschneider et al., 2003)

$$
\psi_{n}=(a n)^{m} \lambda^{n}, \quad a=-\ln \lambda
$$


Inserting Eq.(14) into Eq.(3), the Poisson wavelets $\Psi_{p w}^{(m)}$ read

$$
\Psi_{p w}^{(m)}(\boldsymbol{x}, \boldsymbol{y})=\sum_{n=0}^{\infty}(a n)^{m} \frac{2 n+1}{|\boldsymbol{y}|}\left(\frac{|\boldsymbol{y}|}{|\boldsymbol{x}|}\right)^{n+1} P_{n}\left(\hat{\boldsymbol{x}}^{T} \hat{\boldsymbol{y}}\right) .
$$

Note that the zero-order Poisson wavelet $\Psi_{p w}^{(0)}$ is related to the Poisson kernel $\Psi_{p k}$ as $|\boldsymbol{y}| \Psi_{p w}^{(0)}(\boldsymbol{x}, \boldsymbol{y})=\Psi_{p k}(\boldsymbol{x}, \boldsymbol{y})$. As follows from the spatial representation of the Poisson wavelets

$$
\Psi_{p w}^{(m)}(\boldsymbol{x}, \boldsymbol{y})=\frac{a^{m}}{R}\left(2 \chi^{(m+1)}+\chi^{(m)}\right), \quad \chi^{(m)}=\left(|\boldsymbol{y}| \frac{\partial}{\partial|\boldsymbol{y}|}\right)^{m} \frac{1}{|\boldsymbol{x}-\boldsymbol{y}|},
$$

the zero-order Poisson wavelet $\Psi_{p w}^{(0)}(\boldsymbol{x}, \boldsymbol{y})$ is found to be

$$
\Psi_{p w}^{(0)}(\boldsymbol{x}, \boldsymbol{y})=2|\boldsymbol{y}| \Psi_{r m}^{(1)}(\boldsymbol{x}, \boldsymbol{y})+\Psi_{r m}^{(0)}(\boldsymbol{x}, \boldsymbol{y}) .
$$

Again, the higher-order Poisson wavelets can be computed recursively

$$
\Psi_{p w}^{(m)}(\boldsymbol{x}, \boldsymbol{y})=2|\boldsymbol{y}|^{m+1} \Psi_{r m}^{(m+1)}(\boldsymbol{x}, \boldsymbol{y})+\sum_{k=1}^{m} b_{k, m}|\boldsymbol{y}|^{k} \Psi_{r m}^{(k)}(\boldsymbol{x}, \boldsymbol{y}), \quad m \geq 1,
$$

where the coefficients $b_{k, m}$ of order $m \leq 9$ are provided in Table 1 .

To implement these SRBFs for the gravity observables $\delta g$ and $\Delta g$, the linear observation operators $D_{\delta g}$ and $D_{\Delta g}$ are applied. It is $D_{\delta g}=-\partial / \partial|x|$, and $D_{\Delta g}=D_{\delta g}-2|x|^{-1} \mathfrak{I}$, where $\mathfrak{I}$ is the identity operator. For the spectral representation of the SRBFs, the Legendre coefficients $\psi_{n}$ of degree $n$ are multiplied by the factors $(n+1) /|\boldsymbol{x}|$ and $(n-1) /|\boldsymbol{x}|$, respectively. The corresponding expressions for the spatial representation of the SRBFs are summarized in the Appendix.

The point-mass kernel and the radial multipoles up to the order 5 are shown in Fig. 1. The Poisson kernel is shown in Fig. 2 and the Poisson wavelets up to the order 5 in Fig. 3. The SRBFs are scaled to their maximum values equal to 1 . The depth of all the SRBFs is $10 \mathrm{~km}$ beneath the Bjerhammar sphere $(R=6371 \mathrm{~km})$.

\section{ESTIMATION PRINCIPLE, VARIANCE COMPONENT ESTIMATION, AND REGULARIZATION}

The observation data of different quality are separated into individual observation groups for which the variance factors are estimated using variance component estimation (VCE) techniques (see, e.g., Förstner, 1979; Koch and Kusche, 2002; Kusche, 2003). The same technique is used to determine the regularization parameter. For that reason, the observation equations are formed for $P+1$ observation groups, 
Table 1. Coefficients $\left\{b_{k, m}: k=1, \ldots, m ; m \leq 9\right\}$ to compute Poisson wavelets up to the order 9 according to Eq.(18).

\begin{tabular}{|r|r|r|r|r|r|r|r|r|r|}
\hline$m$ & $k=1$ & $k=2$ & $k=3$ & $k=4$ & $k=5$ & $k=6$ & $k=7$ & $k=8$ & $k=9$ \\
\hline 1 & 3 & & & & & & & & \\
2 & 3 & 8 & & & & & & & \\
3 & 3 & 17 & 13 & & & & & & \\
4 & 3 & 37 & 56 & 21 & & & & & \\
5 & 3 & 77 & 205 & 140 & 31 & & & & \\
6 & 3 & 157 & 692 & 785 & 295 & 43 & & & \\
7 & 3 & 317 & 2233 & 3842 & 2260 & 553 & 57 & & \\
8 & 3 & 637 & 7016 & 17601 & 15142 & 5578 & 952 & 73 & \\
9 & 3 & 1277 & 21685 & 77420 & 93311 & 48610 & 12242 & 1536 & 91 \\
\hline
\end{tabular}

$$
\boldsymbol{l}_{p}+\boldsymbol{\varepsilon}_{p}=\mathbf{A}_{p} \boldsymbol{x}, \quad D\left(\boldsymbol{\varepsilon}_{p}\right)=\mathbf{C}_{p}=\sigma_{p}^{2} \mathbf{W}_{p}^{-1}, \quad p=1, \ldots, P+1,
$$

where $\boldsymbol{l}_{p}$ is the $J_{p} \times 1$ observation vector of observation group $p, \mathbf{A}_{p}$ the $J_{p} \times I$ design matrix, and $\boldsymbol{x}$ the $I \times 1$ vector of local gravity field parameters $\left\{\beta_{i}: i=1, \ldots, I\right\}$. Notice that $\mathbf{A}_{p+1}=\mathbf{I}_{I}, \sigma_{P+1}^{2}=1 / \alpha$, and $\mathbf{W}_{p+1}=\mathbf{R}$, where $\mathbf{I}_{I}$ is the $I \times I$ unit matrix, $\alpha$ is the regularization parameter, and $\mathbf{R}$ the regularization matrix. Assuming that the observation noise is white Gaussian with zero mean, and that the noise variance is the same for the data within each observation group, the variance-covariance matrices $\mathbf{C}_{p}, p=1, \ldots, P$ are scaled unit matrices,

$$
\mathbf{C}_{p}=\sigma_{p}^{2} \mathbf{I}_{J_{p}}, \quad p=1, \ldots, P
$$

where $\mathbf{I}_{J_{p}}$ is the $J_{p} \times J_{p}$ unit matrix, and $\sigma_{p}^{2}$ is the variance factor of observation group $p$.

For known variance factors $\left\{\sigma_{p}^{2}: p=1, \ldots, P+1\right\}$, the least-squares solution of Eq.(19) is

where the normal matrix $\mathbf{N}$ reads

$$
\hat{\boldsymbol{x}}=\mathbf{N}^{-1} \boldsymbol{h}
$$

$$
\mathbf{N}=\sum_{p=1}^{P} \mathbf{N}_{p}+\alpha \mathbf{R}, \quad \mathbf{N}_{p}=\mathbf{A}_{p}^{T} \mathbf{C}_{p}^{-1} \mathbf{A}_{p},
$$

and the right-hand side vector of the normal equations is given by

$$
\boldsymbol{h}=\sum_{p=1}^{P} \boldsymbol{h}_{p}, \quad \boldsymbol{h}_{p}=\mathbf{A}_{p}^{T} \mathbf{C}_{p}^{-1} \boldsymbol{l}_{p}
$$


The Choice of the Spherical Radial Basis Functions in Local Gravity Field Modeling

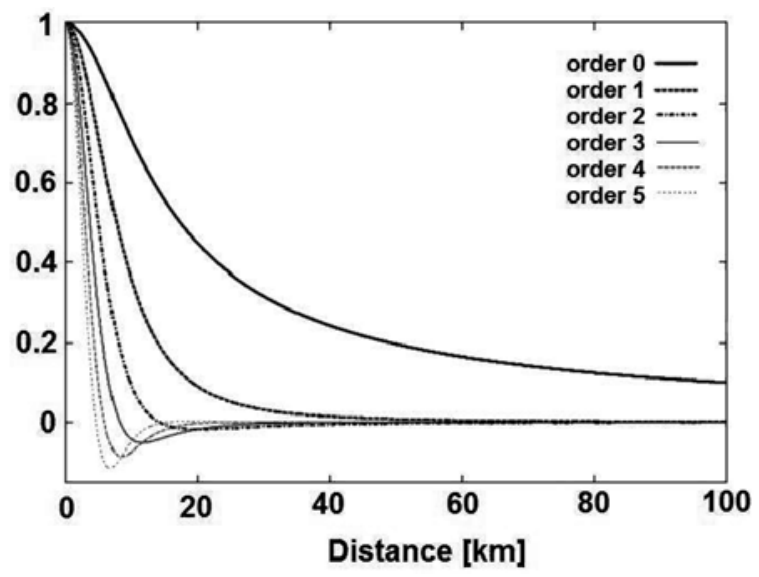

Fig. 1. The radial multipoles of order $0, \ldots, 5($ depth $=10 \mathrm{~km})$. Note that the point-mass kernel is identical to the zero-order radial multipole.

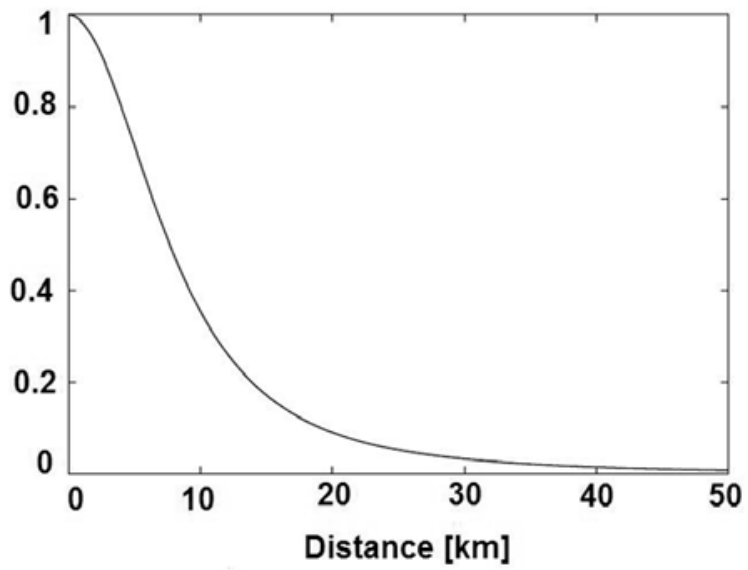

Fig. 2. The Poisson kernel (depth $=10 \mathrm{~km})$.

For unknown variance factors $\sigma_{p}^{2}$, the non-linear optimization problem is solved iteratively. In each iteration step, a least-squares solution, Eq.(22), is computed using the estimated variance factors from the previous step as a-priori variances. New estimates of the variance factors are obtained according to

$$
\sigma_{p}^{2}=\frac{\boldsymbol{\varepsilon}_{p}^{T} \mathbf{W}_{p} \boldsymbol{\varepsilon}_{p}}{r_{p}},
$$

where $r_{p}$ is the redundancy number of observation group $p$,

$$
r_{p}=J_{p}-\operatorname{trace}\left(\mathbf{N}^{-1} \mathbf{N}_{p}\right)
$$




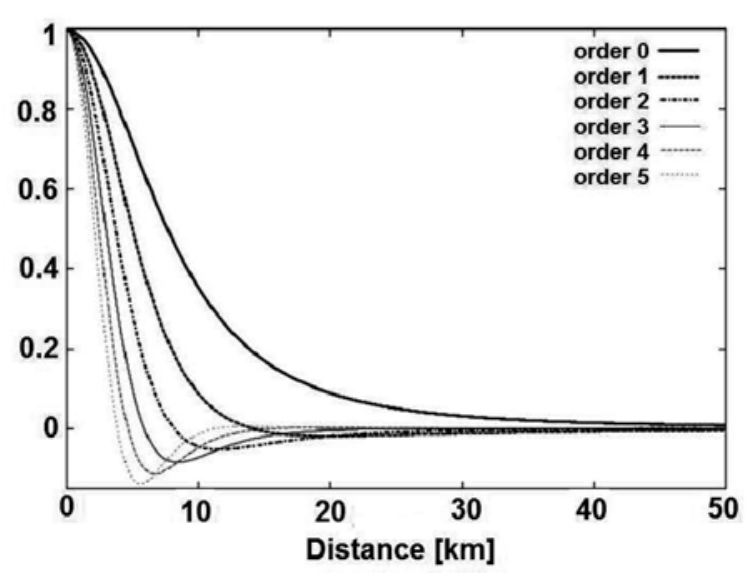

Fig. 3. The Poisson wavelets of order $0, \ldots, 5($ depth $=10 \mathrm{~km})$.

Once the definite variance factors and the associated least-squares solution have been found, the accuracy of the estimated parameters and adjusted observations can be computed from error propagation. The noise variance-covariance matrix of the estimated parameters reads

$$
\mathbf{C}_{\hat{\boldsymbol{x}}}=\left(\sum_{p=1}^{P+1} \mathbf{A}_{p}^{T} \mathbf{C}_{p}^{-1} \mathbf{A}_{p}\right)^{-1}
$$

The noise variance-covariance matrix of the adjusted observations, $\hat{\boldsymbol{l}}=\mathbf{A} \hat{\boldsymbol{x}}$, is given by

$$
\mathbf{C}_{\hat{l}}=\mathbf{A C}_{\hat{\boldsymbol{x}}} \mathbf{A}^{T} \text {. }
$$

\section{RELATION BETWEEN THE DEPTH AND THE CORRELATION LENGTH OF SRBFS}

We define the correlation length of a SRBF as the spherical distance for which the value of the SRBF has dropped to $50 \%$ of its maximal value. For the point-mass kernel and the Poisson kernel, the correlation length is a function of the depth of the SRBF; for the radial multipole of order $\mathrm{m}$ and the Poisson wavelet of order $\mathrm{m}$, the correlation length is a function of the depth and of the order. The relation between the depth (at the interval between 1 and $50 \mathrm{~km}$ ) and the correlation length for the point-mass kernel $\Psi_{p m} \equiv \Psi_{r m}^{(0)}$, the radial multipoles $\Psi_{r m}^{(m)}(m \leq 5)$, the Poisson kernel $\Psi_{p k}$, and the Poisson wavelets $\Psi_{p w}^{(m)}(m \leq 5)$ are shown in Figs. 4-6. This relation is further shown for the SRBFs $D_{\delta g} \Psi$ in Figs. 7-9. 
The relation between depth and correlation length of the investigated SRBF $\Psi$ is well described by a linear function, at least for depth less than $50 \mathrm{~km}$, which covers almost all cases relevant for local gravity field modelling. Moreover, the relation between the depth and the correlation length for the SRBFs $D_{\delta g} \Psi$ and $D_{\Delta g} \Psi$ is very similar.

In addition, we found that the correlation lengths of the Poisson kernel and the zeroorder Poisson wavelet are identical. For the radial multipoles and the Poisson wavelets, the correlation length decreases with increasing order $\mathrm{m}$. The order $m=3$ is used in Section 5. The correlation lengths of $D_{\delta g} \Psi_{p m}, D_{\Delta g} \Psi_{p m}$, and $\Psi_{p k}$ are very similar, which can be expected from the analytical expressions.

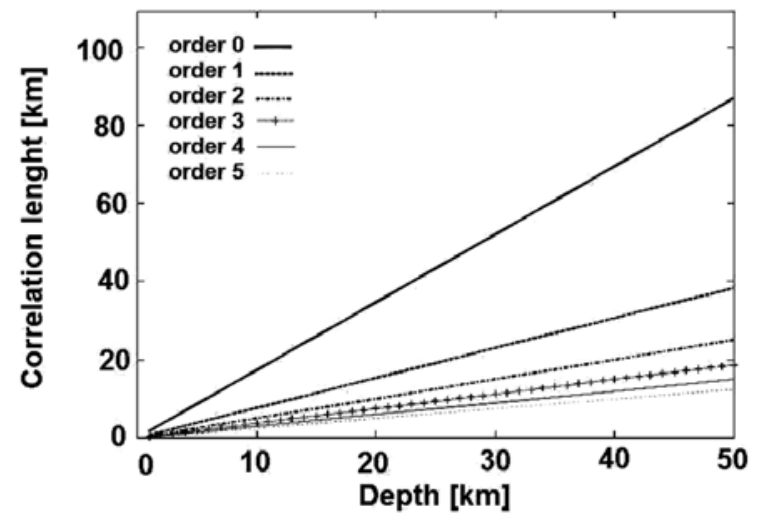

Fig. 4. The relation between the depth (at the interval between 1 and $50 \mathrm{~km}$ ) and the correlation length for the point-mass kernel $\Psi_{p m} \equiv \Psi_{r m}^{(0)}$ and the radial multipoles $\Psi_{r m}^{(m)}(m \leq 5)$.

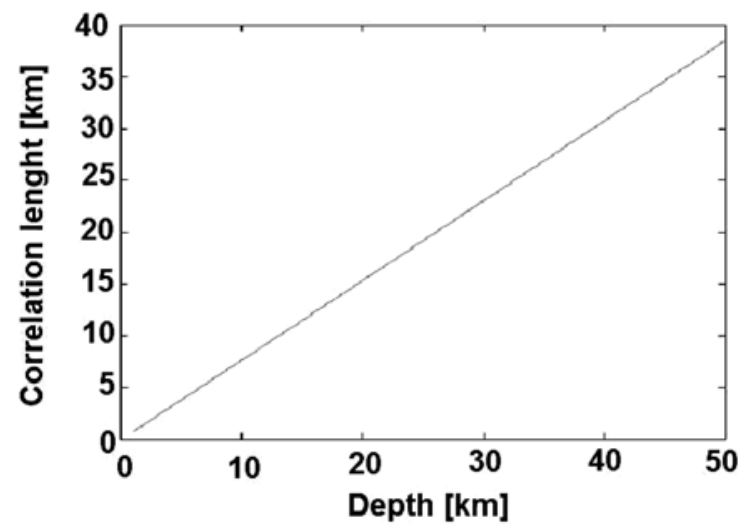

Fig. 5. The relation between the depth (at the interval between 1 and $50 \mathrm{~km}$ ) and the correlation length for the Poisson kernel $\Psi_{p k}$. 


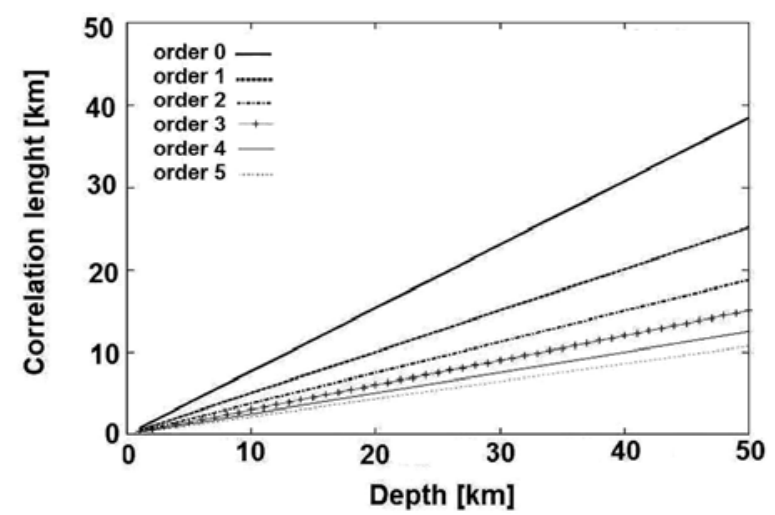

Fig. 6. The relation between the depth (at the interval between 1 and $50 \mathrm{~km}$ ) and the correlation length for the Poisson wavelets $\Psi_{p w}^{(m)}(m \leq 5)$.

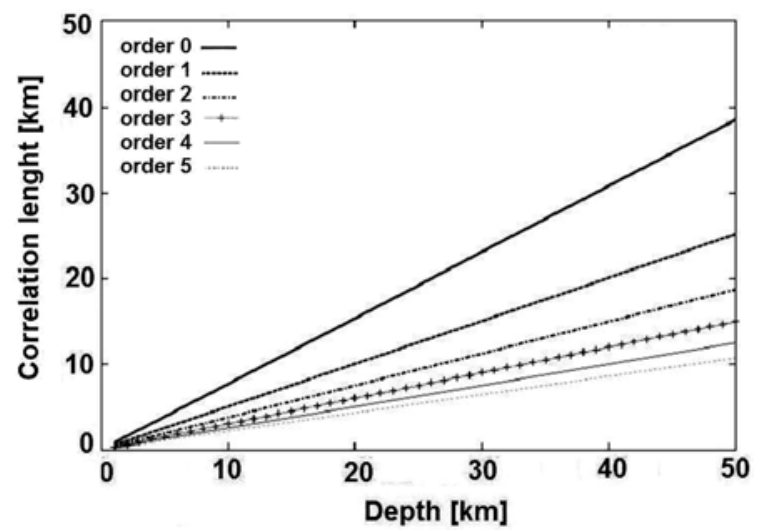

Fig. 7. The relation between the depth (at the interval between 1 and $50 \mathrm{~km}$ ) and the correlation length for the SRBFs $D_{\delta g} \Psi_{p m} \equiv D_{\delta g} \Psi_{r m}^{(0)}$ and $D_{\delta g} \Psi_{r m}^{(m)}(m \leq 5)$.

\section{NUMERICAL STUDY}

To investigate the performance of the SRBFs in local gravity field modelling, they have been applied to real terrestrial gravity anomalies over the Netherlands. The following questions will be addressed: (i) does the quality of the gravity field model depend on the type of SRBF? (ii) are there differences between the optimal depths and/or the correlation lengths for the various types of SRBFs? (iii) how does RMS minimization perform compared with GCV for the choice of the optimal depth?

30178 free-air gravity anomalies have been used, which cover the Netherlands and surrounding areas. From them, 653 have been selected randomly to serve as control points for the RMS minimization of the differences between observed and predicted values for optimal depth selection. The data have been grouped into 7 observation groups, depending 
on a priori information about the expected accuracy, the data type (point values or gridded values) and the location. The data over the Netherlands are assigned to 3 observation groups. The EIGEN-CG03c global gravity field model has been subtracted from the data. No topographic corrections were applied due to the flatness of the area. 5628 SRBFs have been used to parameterize the local gravity field, which is about $19 \%$ of the number of observations. We found that when adding more SRBFs, the quality of the gravity field solution does not improve or even gets worse as more and more data noise is modelled. The mean distance between the SRBFs is about $4.3 \mathrm{~km}$. We found that additional SRBFs should be located outside the target area within a distance of about 3 times the correlation length of the SRBF. The same holds for the data area relative to the area of SRBF parameterization. For more details about the input data, the choice of the SRBF locations, and the data processing methodology, we refer to Klees et al. (2008).

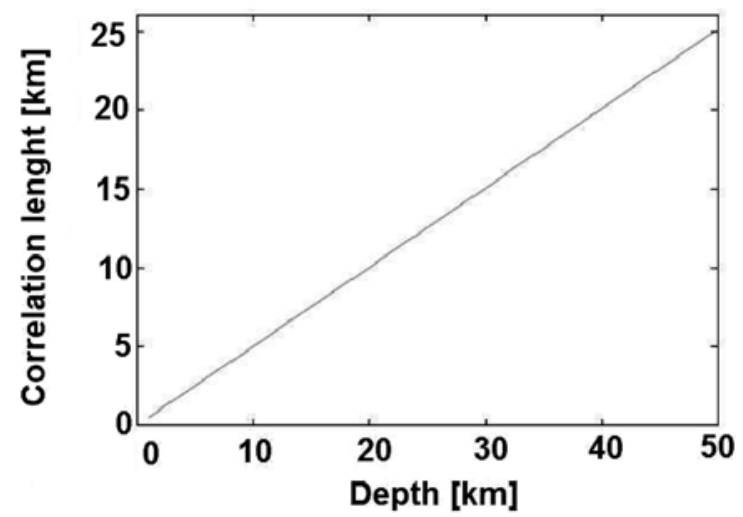

Fig. 8. The relation between the depth (at the interval between 1 and $50 \mathrm{~km}$ ) and the correlation length of $D_{\delta g} \Psi_{p k}$.

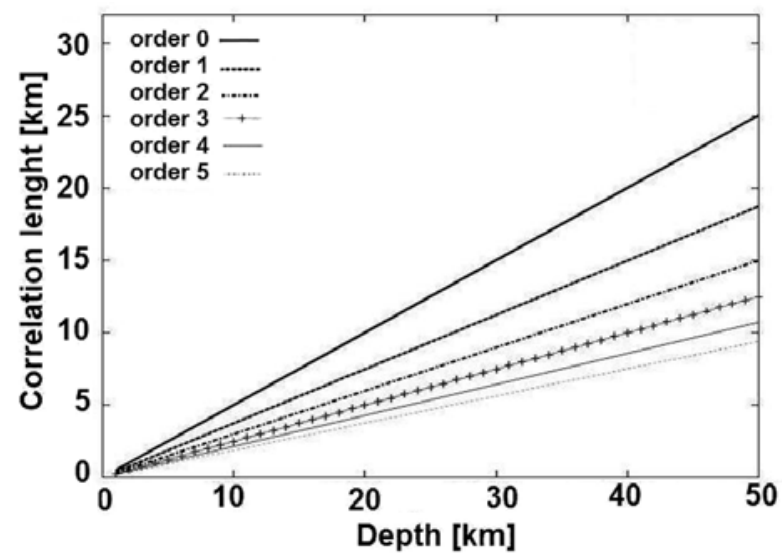

Fig. 9. The relation between the depth (at the interval between 1 and $50 \mathrm{~km}$ ) and the correlation length for the SRBF $D_{\delta g} \Psi_{p w}^{(m)}(m \leq 5)$. 
GCV is used to select the optimal depth $d$ of the SRBFs. The GCV objective functional

$$
\Phi_{G C V}(d)=\arg \min \sum_{j=1}^{J} \frac{J\left(\hat{l}_{j}(d)-l_{j}\right)^{2}}{(\operatorname{trace}(\mathbf{I}-\mathbf{Q}(d)))^{2}}
$$

is shown as function of the depth in Figs. 10-13, where $\mathbf{Q}$ is the influence matrix defined as $\mathbf{Q} \boldsymbol{l}=\mathbf{A} \hat{\boldsymbol{x}}$, and $J=\sum_{p=1}^{P} J_{p}$ is the total number of observations. The optimal depth depends significantly on the type of the SRBF. The point-mass kernel has the shallowest optimal depth of $10.5 \mathrm{~km}$; the largest optimal depth, $23 \mathrm{~km}$, is found for the Poisson wavelet of order 3 . The GCV objective functional behaves relatively flat around the optimal depth, which means that there is a range of depths very close to the optimal one. For instance, for the Poisson wavelet the range is $21-25 \mathrm{~km}$. We computed gravity field solutions using all depth over this range and found that the differences between them are statistically insignificant. Therefore, the gravity field solution is somehow robust with respect to the choice of the depth; fixing the depth with an accuracy of a few kilometres is sufficient for the data set used in this study.
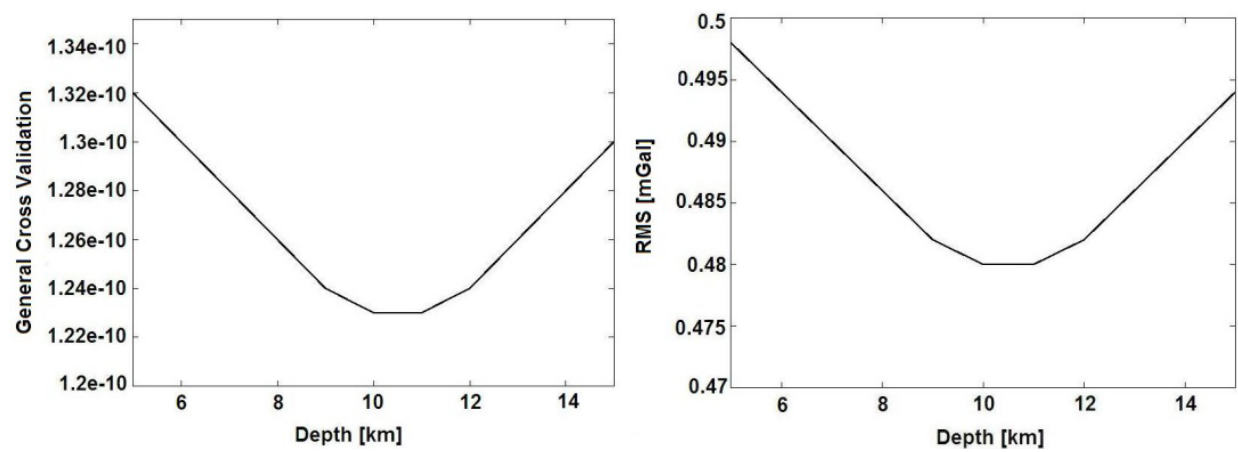

Fig. 10. GCV (left) and RMS minimization (right) for the choice of the optimal depth of the pointmass kernel. A search interval between $5 \mathrm{~km}$ and $15 \mathrm{~km}$ with a step of $1 \mathrm{~km}$ was used.
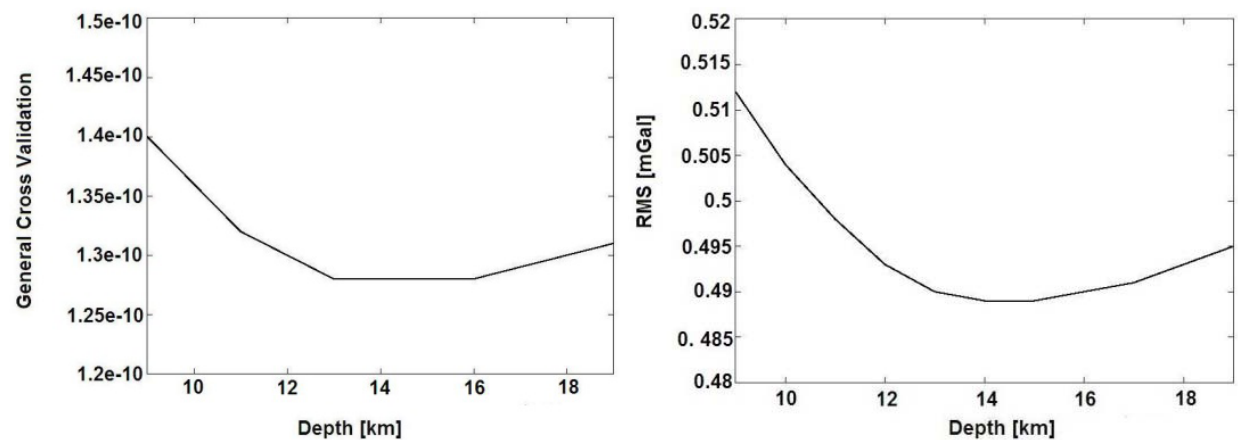

Fig. 11. GCV (left) and RMS minimization (right) for the choice of the optimal depth of the radial multipole of order 3. A search interval between $9 \mathrm{~km}$ and $19 \mathrm{~km}$ with a step of $1 \mathrm{~km}$ was used. 
The results of GCV were verified by the RMS minimization technique at the control points within the target area. As follows from the comparison of GCV and RMS minimization (see Figs. 10-13), both techniques provide very similar results. Specifically, the optimal depth between 10-11 km for the point-mass kernel can be recognized from both techniques. The optimal depth for the radial multipole of order 3 is about $13-16 \mathrm{~km}$ (GCV) or 14-15 km (RMS minimization). For the Poisson kernel the optimal depth is approximately between $11-14 \mathrm{~km}$, and between $21-25 \mathrm{~km}$ for the Poisson wavelet of order 3.

Table 2 shows that the RMS of the least-squares residuals is about the same for all SRBFs provided that the depth is chosen optimally; the differences are below $10 \mu \mathrm{Gal}$, which is significantly smaller than accuracy of the gravity anomalies $(\sim 0.5 \mathrm{mGal}$, cf. Table 2). Therefore, we conclude that all investigated SRBFs yield about the same quality of the gravity field solution for the data set used in this study.

The SRBFs for the optimal depths are shown in Fig. 14. The SRBFs have different correlation lengths, varying between $4 \mathrm{~km}$ (radial multipole of order 3 ) and $8.5 \mathrm{~km}$ (pointmass kernel). This variation is significantly smaller than the variation of the optimal depths, which is also clearly visible in Figs. 7-9. Moreover, the corresponding correlation
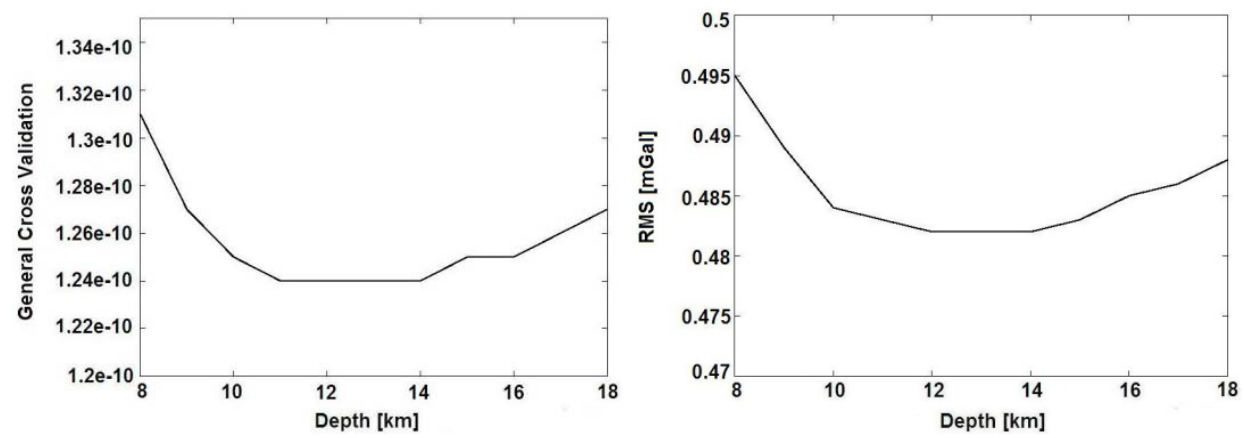

Fig. 12. GCV (left) and RMS minimization (right) for the choice of the optimal depth of the Poisson kernel. A search interval between $8 \mathrm{~km}$ and $18 \mathrm{~km}$ with a step of $1 \mathrm{~km}$ was used.
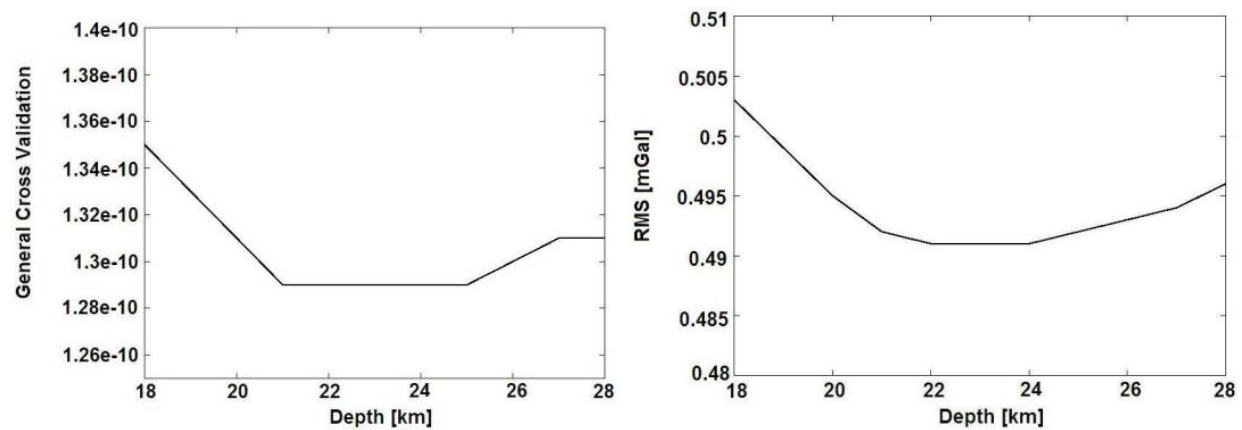

Fig. 13. GCV (left) and RMS minimization (right) for the choice of the optimal depth of the Poisson wavelet of order 3 . A search interval between $18 \mathrm{~km}$ and $28 \mathrm{~km}$ with a step of $1 \mathrm{~km}$ was used. 


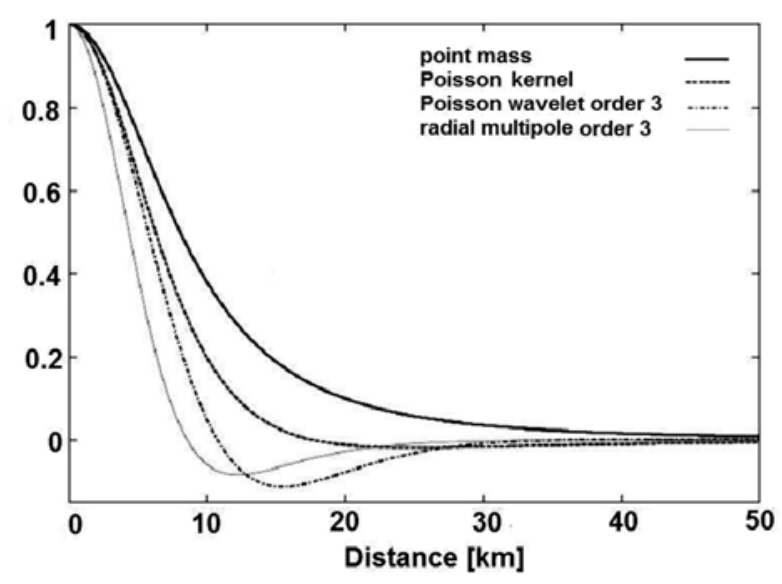

Fig. 14. The SRBFs $D_{\Delta g} \Psi$ at the optimal depths (point-mass kernel: $10.5 \mathrm{~km}$, radial multipole of order 3: $14.5 \mathrm{~km}$, Poisson kernel: $12.5 \mathrm{~km}$, Poisson wavelet of order 3: $23 \mathrm{~km}$ ).

lengths of the functions $D_{\Delta g} \Psi$ are much smaller than the correlation length of the empirical gravity anomaly auto-covariance function, which is about $17 \mathrm{~km}$.

To investigate the influence of the correlation length on the quality of the gravity field solution, least-squares solutions have been computed for the extreme correlation lengths $4 \mathrm{~km}$ and $8 \mathrm{~km}$ for each type of SRBF. The quality of the solution has been quantified in terms of the RMS difference between predicted and observed gravity anomalies at the control points. The results are summarized in Tables 3 and 4 . They reveal that a change of the correlation length over the range $4-8 \mathrm{~km}$ changes the RMS difference by less than $32 \mu \mathrm{Gal}$. This is below the estimated accuracy of the gravity anomalies.

The correlation length for a particular type of SRBF indicates to what extent the data area must be larger than the target area. From our experiment, the largest data extension outside the target area is needed when the point-mass kernel is used (correlation length $8.5 \mathrm{~km}$ ), while the smallest extension is needed for the radial multipole of order 3 (correlation length $4 \mathrm{~km}$ ).

\section{CONCLUSIONS}

The proper choice of the SRBF in local gravity field modelling from terrestrial gravity data was investigated using real data. A penalized least-squares technique was applied to estimate the gravity field parameters. The main result of the study is that for each type of SRBF we obtain comparable accuracies if the depth of SRBF is chosen optimally.

GCV and RMS minimization techniques were applied for the selection of the optimal depth. We demonstrated that both methods provide very similar results. Moreover, the gravity field solution does not change significantly if the depth is changed over a range of several $\mathrm{km}$. 
Table 2 The optimal depth of $\Psi$, the correlation length of $D_{\delta g} \Psi$, and the RMS of the least-squares residuals for different types of SRBFs.

\begin{tabular}{|l|c|c|c|}
\hline \multicolumn{1}{|c|}{ Type of SRBF } & Optimal Depth $[\mathrm{km}]$ & Correlation Length $[\mathrm{km}]$ & RMS [mGal] \\
\hline Point-mass kernel & 10.5 & 8.0 & 0.48 \\
Radial multipole of order 3 & 14.5 & 4.2 & 0.49 \\
Poisson kernel & 12.5 & 6.2 & 0.48 \\
Poisson wavelet of order 3 & 23.0 & 5.5 & 0.49 \\
\hline
\end{tabular}

Table 3. RMS differences between predicted and observed gravity anomalies at the control points for 4-km correlation length of the SRBFs $D_{\Delta g} \Psi$.

\begin{tabular}{|l|c|c|c|}
\hline \multicolumn{1}{|c|}{ Type of SRBF } & Correlation Length $[\mathrm{km}]$ & Depth $[\mathrm{km}]$ & RMS [mGal] \\
\hline Point-mass kernel & 4 & 5.5 & 0.507 \\
Radial multipole of order 3 & 4 & 14 & 0.490 \\
Poisson kernel & 4 & 8 & 0.495 \\
Poisson wavelet of order 3 & 4 & 16 & 0.509 \\
\hline
\end{tabular}

Table 4. RMS differences between predicted and observed gravity anomalies at a set of control points for 8-km correlation length of the SRBFs $D_{\Delta g} \Psi$.

\begin{tabular}{|l|c|c|c|}
\hline \multicolumn{1}{|c|}{ Type of SRBF } & Correlation Length $[\mathrm{km}]$ & Depth $[\mathrm{km}]$ & RMS [mGal] \\
\hline Point-mass kernel & 8 & 11 & 0.482 \\
Radial multipole of order 3 & 8 & 28 & 0.512 \\
Poisson kernel & 8 & 17 & 0.485 \\
Poisson wavelet of order 3 & 8 & 34 & 0.506 \\
\hline
\end{tabular}

The correlation lengths for the different types of SRBFs at the optimal depth were compared. The correlation length determines the extension of the data area relative to the target area. The comparison indicates that the point-mass kernel requires the largest data area.

\section{APPENDIX A}

\section{ANALYTICAL EXPRESSIONS FOR FUNCTIONALS OF THE SRBFS}

For the point-mass kernel functionals $D_{\delta g} \Psi_{p m}$ and $D_{\Delta g} \Psi_{p m}$, we find

$$
D_{\delta g} \Psi_{p m}(\boldsymbol{x}, \boldsymbol{y})=\frac{|\boldsymbol{x}|-|\boldsymbol{y}|\left(\hat{\boldsymbol{x}}^{T} \hat{\boldsymbol{y}}\right)}{|\boldsymbol{x}-\boldsymbol{y}|^{3}},
$$




$$
D_{\Delta g} \Psi_{p m}(\boldsymbol{x}, \boldsymbol{y})=D_{\delta g} \Psi_{p m}(\boldsymbol{x}, \boldsymbol{y})-\frac{2}{|\boldsymbol{x}|} \Psi_{p m}(\boldsymbol{x}, \boldsymbol{y}) .
$$

For the radial multipole of order 0 follows

$$
\begin{aligned}
& D_{\delta g} \Psi_{r m}^{(0)}(\boldsymbol{x}, \boldsymbol{y}) \equiv D_{\delta g} \Psi_{p m}(\boldsymbol{x}, \boldsymbol{y}), \\
& D_{\Delta g} \Psi_{r m}^{(0)}(\boldsymbol{x}, \boldsymbol{y}) \equiv D_{\Delta g} \Psi_{p m}(\boldsymbol{x}, \boldsymbol{y}) .
\end{aligned}
$$

For the radial multipole of order 1, we find

$$
\begin{gathered}
D_{\delta g} \Psi_{r m}^{(1)}(\boldsymbol{x}, \boldsymbol{y})=-\frac{\left(\hat{\boldsymbol{x}}^{T} \hat{\boldsymbol{y}}\right)}{|\boldsymbol{x}-\boldsymbol{y}|^{3}}+\frac{3}{|\boldsymbol{x}-\boldsymbol{y}|^{5}}\left[|\boldsymbol{x}|\left(\hat{\boldsymbol{x}}^{T} \hat{\boldsymbol{y}}\right)-|\boldsymbol{y}|\right]\left[|\boldsymbol{x}|-|\boldsymbol{y}|\left(\hat{\boldsymbol{x}}^{T} \hat{\boldsymbol{y}}\right)\right] \\
D_{\Delta g} \Psi_{r m}^{(1)}(\boldsymbol{x}, \boldsymbol{y})=D_{\delta g} \Psi_{r m}^{(1)}(\boldsymbol{x}, \boldsymbol{y})-\frac{2}{|\boldsymbol{x}|} \Psi_{r m}^{(1)}(\boldsymbol{x}, \boldsymbol{y}) .
\end{gathered}
$$

The higher- order $(m \geq 2)$ radial multipoles can be computed recursively from

$$
\begin{gathered}
D_{\delta g} \Psi_{r m}^{(\mathrm{m})}(\boldsymbol{x}, \boldsymbol{y})=-(2 m-1) \frac{|\boldsymbol{x}|-|\boldsymbol{y}|\left(\hat{\boldsymbol{x}}^{T} \hat{\boldsymbol{y}}\right)}{|\boldsymbol{x}-\boldsymbol{y}|} \Psi_{r m}^{(m-1)}(\boldsymbol{x}, \boldsymbol{y}) \Psi_{r m}^{(1)}(\boldsymbol{x}, \boldsymbol{y}) \\
+(2 m-1)|\boldsymbol{x}-\boldsymbol{y}|\left(\Psi_{r m}^{(m-1)}(\boldsymbol{x}, \boldsymbol{y}) D_{\delta g} \Psi_{r m}^{(1)}(\boldsymbol{x}, \boldsymbol{y})+\Psi_{r m}^{(1)}(\boldsymbol{x}, \boldsymbol{y}) D_{\delta g} \Psi_{r m}^{(m-1)}(\boldsymbol{x}, \boldsymbol{y})\right) \\
-(m-1)^{2}\left(2 \Psi_{r m}^{(0)}(\boldsymbol{x}, \boldsymbol{y}) \Psi_{r m}^{(m-2)}(\boldsymbol{x}, \boldsymbol{y}) D_{\delta g} \Psi_{r m}^{(0)}(\boldsymbol{x}, \boldsymbol{y})\right. \\
\left.+\Psi_{r m}^{(0)}(\boldsymbol{x}, \boldsymbol{y}) \Psi_{r m}^{(0)}(\boldsymbol{x}, \boldsymbol{y}) D_{\delta g} \Psi_{r m}^{(m-2)}(\boldsymbol{x}, \boldsymbol{y})\right), \quad m \geq 2, \\
D_{\Delta g} \Psi_{r m}^{(m)}(\boldsymbol{x}, \boldsymbol{y})=D_{\delta g} \Psi_{r m}^{(m)}(\boldsymbol{x}, \boldsymbol{y})-\frac{2}{|\boldsymbol{x}|} \Psi_{r m}^{(m)}(\boldsymbol{x}, \boldsymbol{y}) .
\end{gathered}
$$

The expressions for the Poisson kernel functionals $D_{\delta g} \Psi_{p k}$ and $D_{\Delta g} \Psi_{p k}$ are given by

$$
\begin{gathered}
D_{\delta g} \Psi_{p k}(\boldsymbol{x}, \boldsymbol{y})=-|\boldsymbol{y}|\left[\frac{2|\boldsymbol{x}|^{3}}{|\boldsymbol{x}-\boldsymbol{y}|^{3}}-\frac{3\left(|\boldsymbol{x}|^{2}-|\boldsymbol{y}|^{2}\right)\left(|\boldsymbol{x}|-|\boldsymbol{y}|\left(\hat{\boldsymbol{x}}^{T} \hat{\boldsymbol{y}}\right)\right)}{|\boldsymbol{x}-\boldsymbol{y}|^{5}}\right] \\
D_{\Delta g} \Psi_{p k}(\boldsymbol{x}, \boldsymbol{y})=D_{\delta g} \Psi_{p k}(\boldsymbol{x}, \boldsymbol{y})-\frac{2}{|\boldsymbol{x}|} \Psi_{p k}(\boldsymbol{x}, \boldsymbol{y}) .
\end{gathered}
$$


The expressions for the zero-order Poisson wavelet functionals $D_{\delta g} \Psi_{p w}^{(0)}$ and $D_{\Delta g} \Psi_{p w}^{(0)}$ are related to the expressions for the Poisson kernel functionals $D_{\delta g} \Psi_{p k}$ and $D_{\Delta g} \Psi_{p k}$, Eqs. (I.9) and (I.10), as follows

$$
\begin{gathered}
D_{\delta g} \Psi_{p w}^{(0)}(\boldsymbol{x}, \boldsymbol{y})=|\boldsymbol{y}|^{-1} D_{\delta g} \Psi_{p k}(\boldsymbol{x}, \boldsymbol{y}) \\
D_{\Delta g} \Psi_{p w}^{(0)}(\boldsymbol{x}, \boldsymbol{y})=D_{\delta g} \Psi_{p w}^{(0)}(\boldsymbol{x}, \boldsymbol{y})-\frac{2}{|\boldsymbol{x}|} \Psi_{p w}^{(0)}(\boldsymbol{x}, \boldsymbol{y}) .
\end{gathered}
$$

The higher-order Poisson wavelets can be obtained recursively from

$$
\begin{gathered}
D_{\delta g} \Psi_{p w}^{(m)}(\boldsymbol{x}, \boldsymbol{y})=-2|\boldsymbol{y}|^{m+1} D_{\delta g} \Psi_{r m}^{(m+1)}(\boldsymbol{x}, \boldsymbol{y})-\sum_{k=1}^{m} b_{k, m}|\boldsymbol{y}|^{k} D_{\delta g} \Psi_{r m}^{(k)}(\boldsymbol{x}, \boldsymbol{y}), \\
m \geq 1, \\
D_{\Delta g} \Psi_{p w}^{(m)}(\boldsymbol{x}, \boldsymbol{y})=D_{\delta g} \Psi_{p w}^{(m)}(\boldsymbol{x}, \boldsymbol{y})-\frac{2}{|\boldsymbol{x}|} \Psi_{p w}^{(m)}(\boldsymbol{x}, \boldsymbol{y}) .
\end{gathered}
$$

\section{References}

Blaha G., Blessette R.P. and Hadgigeorge G., 1986. Global point-mass adjustment of the oceanic geoid based on satellite altimetry. Marine Geodesy, 10, 97-129.

Chambodut A., Panet I., Mandea M., Diament M., Holschneider M. and Jamet O., 2005. Wavelet frames: an alternative to spherical harmonic representation of potential fields. Geophys. J. Int., 163, 875-899.

Förstner W., 1979. Ein Varfahren zur Schätzung von Variaz- und Kovarianzkomponenten. Allgemeine Vermessungs-Nachrichten, 86, 446-453.

Freeden W., Gervens T. and Schreiner M., 1998. Constructive Approximation of the Sphere (with Applications to Geomathematics). Oxford Science Publication, Clarendon Press.

Hardy R.L. and Göpfert W.M., 1975. Least squares prediction of gravity anomalies, geoidal undulations, and deflections of the vertical with multiquadric harmonic functions. Geophys. Res. Lett., 2, 423-426.

Heikkinen M., 1981. Solving the Shape of the Earth by Using Digital Density Models. Report 81(2), Finnish Geodetic Institute, Helsinki, Finland.

Holschneider M., Chambodut A. and Mandea M., 2003. From global to regional analysis of the magnetic field on the sphere using wavelet frames. Phys. Earth Planet. Inter., 135, 107-124.

Klees R. and Wittwer T., 2007. A data-adaptive design of a spherical basis function network for gravity field modeling. In: Tregoning P. and Rizos C. (Eds.), Dynamic Planet - Monitoring and Understanding a Dynamic Planet with Geodetic and Oceanographic Tools. IAG Symposia, 130, Springer-Verlag, Berlin, Heidelberg, 303-328.

Klees R., Tenzer R., Prutkin I. and Wittwer T., 2008. A data-driven approach to local gravity field modeling using spherical radial basis functions. J. Geodesy, 82, 457-471, doi: 10.1007/s00190-007-0196-3. 
Koch K.R. and Kusche J., 2002. Regularization of geopotential determination from satellite data by variance components. J. Geodesy, 76, 259-268.

Krarup T., 1969. A Contribution to the Mathematical Foundation of Physical Geodesy. Report 44, Danish Geodetic Institute, Copenhagen, Denmark.

Kusche J., 2003. A Monte-Carlo technique for weight estimation in satellite geodesy. J. Geodesy, 76, 641-652.

Lehmann R., 1993. The method of free-positioned point masses - geoid studies on the Gulf of Bothnia. Bulletin Géodésique, 67, 31-40.

Lehmann R., 1995. Gravity field approximation using point masses in free depths. International Association of Geodesy Buleltin, Section IV, No.1, 129-140.

Lelgemann D., 1981. On numerical properties of interpolation with harmonic kernel functions. Manuscripta Geodaetica, 6, 157-191.

Lelgemann D. and Marchenko A.N., 2001. On Concepts for Modeling the Earth's Gravity Field. Deutsche Geodätische Kommission bei der Bayerischen Akademie der Wissenschaften, Reihe A, Heft Nr. 117, München, Germany.

Marchenko A.N., 1998. Parameterization of the Earth's Gravity Field: Point and Line Singularities. Lviv Astronomical and Geodetical Society, Lviv, Ukraine.

Panet I., Chambodut A., Panet I., Diament M., Holschneider M. and Jamet O., 2006. New insights on intraplate volcanism in French Polynesia from wavelet analysis of GRACE, CHAMP, and sea surface data. J. Geophys. Res., 111, B09403, doi: 10.1029/2005JB004141.

Reilly J.P. and Herbrechtsmeier E.H., 1978. A systematic approach to modeling the geopotential with point mass anomalies. J. Geophys. Res., 83, 841-844.

Sansò F. and Tscherning C.C., 2003. Fast spherical collocation: theory and examples. J. Geodesy, 77, 101-112.

Schmidt M., Kusche J., van Loon J., Shum C.K., Han S.C. and Fabert O., 2005. Multi-resolution representation of regional gravity data. In: Jekeli C., Bastos L. and Fernandes J. (Eds.), Gravity, Geoid and Space Missions. Springer-Verlag, Berlin, Heiderlberg, New York, 167-172.

Schmidt M., Fengler M., Mayer-Gürr T., Eicker A., Kusche J., Sanchez J. and Han S.C., 2007. Regional gravity modelling in terms of spherical base functions. J. Geodesy, 8, 17-38, doi: 10.1007/s00190-006-0101-5.

Sünkel H., 1981. Point Mass Models and the Anomalous Gravitational Field. Report No. 328, The Ohio State University, Dept of Geod Sciences, Columbus, Ohio.

Sünkel H., 1983. The Generation of a Mass Point Model from Surface Gravity Data. Report No. 353, The Ohio State University, Dept of Geod Sciences, Columbus, Ohio.

Tscherning C.C., 1986. Functional methods for gravity field approximation. In: Sünkel H. (Ed.), Mathematical and Numerical Techniques in Physical Geodesy. Lecture Notes in Earth Sciences, 7, Springer-Verlag, Berlin, Heidelberg, 3-47.

Vermeer M., 1992. Geoid determination with mass point frequency domain inversion in the Mediterranean. Mare Nostrum, GEOMED Report 2, Madrid, 109-119.

Vermeer M., 1995. Mass point geopotential modeling using fast spectral techniques; historical overview, toolbox description, numerical experiment. Manuscripta Geodaetica, 20, 362-378. 